Check for updates

Cite this: RSC Adv., 2019, 9, 24987

Received 26th June 2019

Accepted 29th July 2019

DOI: $10.1039 / c 9 r a 04814 f$

rsc.li/rsc-advances

\title{
Tumor homing peptide modified liposomes of capecitabine for improved apoptotic activity and HER2 targeted therapy in breast cancer: in vitro studies
}

\author{
Mantosh Kumar Singh, (DD a Sai Kiran S. S. Pindiprolu, (DD b Bharat Kumar Reddy \\ Sanapalli, ${ }^{a}$ Vidyasrilekha Yele ${ }^{c}$ and G. N. K. Ganesh*a
}

In the present study, we have formulated a liposomal formulation of cytotoxic agent capecitabine (CAP) to overcome its bioavailability issues. Then we have surface modified CAP loaded liposomes (CAP-LPS) with a tumour homing peptide (THP-CAP-LPs) to achieve site specific delivery to breast cancer cells. We found a significant cellular internalization of THP-CAP-LPs when compared to unmodified CAP-LPS. The cytotoxic effect of CAP was also significantly improved with THP-CAP-LPs by downregulating antiapoptotic proteins and upregulating pro-apoptotic proteins as observed by Western blot analysis. THPCAP-LPs mediated delivery of CAP can be, therefore, a promising approach for improving antitumor activity and reducing off-target effects.
\end{abstract}

\section{Introduction}

Based on the expression of estrogen receptor (ER), progesterone receptor (PR) and human epidermal growth factor receptor-2 (HER2) receptor, breast cancers can be classified into estrogen receptor positive (luminal subtype), HER2, PR and triplenegative breast cancer (TNBC), which lacks ER, PR and HER2 expression. ${ }^{1-3}$ The HER2 receptor has an intracellular tyrosine kinase domain and an extracellular ligand binding domain, and is involved in important stages of growth and cell differentiation. As a key gene of cell survival, HER2 overexpression can lead to malignant progression, which is associated with unfavourable prognosis of breast, ovary, gastric, prostate cancers, among others. HER 2 overexpression is present in approximately $25 \%$ of all breast cancers and usually is associated with more aggressive disease and endocrine therapy resistance. Studies showed that the inhibition of HER2 expression induced significant apoptosis in breast cancer cells and therefore HER2 became a target.

5-Fluoro Uracil (5-FU) is a commonly used drug for treatment of HER2 positive breast cancer. Capecitabine (CAP) is a prodrug of 5FU and effective in treatment of breast cancer. However, off target effects and poor bioavailability are the major limitations for CAP based therapy. Drug delivery using liposomes is a widely

${ }^{a}$ Department of Pharmaceutics, JSS College of Pharmacy, Ooty, JSS Academy of Higher Education \& Research, India. E-mail: gnk@jssuni.edu.in

${ }^{b}$ Department of Pharmacology, JSS College of Pharmacy, Ooty, JSS Academy of Higher Education \& Research, India

${ }^{c}$ Department of Pharmaceutical Chemistry, JSS College of Pharmacy, Ooty, JSS Academy of Higher Education \& Research, India recognized approach for minimizing off-target effects and to increase the concentration of drug at the tumour site. Liposomes with appropriate stealth properties, avoid rapid clearance and accumulate at the site of tumour by enhanced permeation-retention (EPR) effect. ${ }^{4,5}$ However, compelling body of evidence suggest that this accumulation does not provide specific targeting and sufficient intracellular delivery. ${ }^{6}$ The concept of active targeting approach by conjugating an appropriate peptide for specific tumour marker on the surface of nanocarriers is an attractive strategy for selective delivery of drugs to tumours. ${ }^{7}$ In the present study we, therefore, propose HER2+ breast cancer cell specific binding or tumour homing peptide (THP) (WNLPWYYSVSPTC) conjugated CAP liposomes (THP-CAP-LPs) for site specific targeting and improved cell uptake of CAP in breast cancer cells.

\section{Results \& discuss}

\section{Optimization of THP-CAP-LPs}

The optimization was done based on the responses observed for all 20 formulations (Table 2). The effects of pre-determined factors on particle size (PS) and entrapment efficiency (EE) was observed using perturbation plots and response surface plots (Fig. 1). The model was validated by applying analysis of variance (ANOVA) ${ }^{\mathbf{2 0}}$ (Tables 3 and 4 ). The optimized formulation was selected using desirability criterion of PS and EE. It was predicted according to the design that $445.44 \mathrm{mg}$ of cholesterol acrylate, $43.05 \mathrm{mg}$ of CAP and $5.01 \mathrm{~min}$ of sonication will give PS of $114.036 \mathrm{~nm}$ and $\mathrm{EE}$ of $80.87 \%$ (Fig. 2). Based on the predicted composition of lipid surfactant and co-surfactant a new batch of 
Table 1 Independent and dependent variables

\begin{tabular}{llrl}
\hline & \multicolumn{2}{l}{ Levels } & \\
\cline { 2 - 3 } Factors & Low $(-1)$ & Medium (0) & High (+1) \\
\hline Independent variables & & & \\
$X 1$ (mg) (THP-CL) & 262.2 & 500 & 737.8 \\
$X 2$ (mg) (CAP) & 16.1 & 25 & 33.9 \\
$X 3$ (min) (sonication time) & 3 & 6 & 9 \\
& & & \\
Dependent variables & & & \\
$Y 1$ (particle size) & Minimize & & \\
$Y 2$ (entrapment efficiency) & Maximize & & \\
& & &
\end{tabular}

Table 2 Optimization of THP-CAP-LPs using central composite design (CCD)

\begin{tabular}{lllllll}
\hline & & & Factor 2 & Factor 3 & $\begin{array}{l}\text { Response } \\
1 Y 1\end{array}$ & $\begin{array}{l}\text { Response } \\
2 Y 2\end{array}$ \\
\hline Std & Run & Factor 1 A:X1 & B:X2 & C:X3 & 132.6 & \\
1 & 1 & 262.2 & 32.2 & 3 & 200.9 & 63.6 \\
20 & 2 & 500 & 50 & 6 & 104.32 & 82.1 \\
7 & 3 & 262.2 & 67.8 & 9 & 215.92 & 67.2 \\
19 & 4 & 500 & 50 & 6 & 104.31 & 85.2 \\
18 & 5 & 500 & 50 & 6 & 104.3 & 79.3 \\
12 & 6 & 500 & 79.9 & 6 & 205.45 & 66 \\
8 & 7 & 737.8 & 67.8 & 9 & 194.22 & 64.7 \\
5 & 8 & 262.2 & 32.2 & 9 & 188.74 & 59.3 \\
15 & 9 & 500 & 50 & 6 & 104.26 & 82.8 \\
11 & 10 & 500 & 20.0 & 6 & 181.92 & 68.2 \\
4 & 11 & 737.8 & 67.8 & 3 & 202.44 & 63.9 \\
13 & 12 & 500 & 50 & 0.9 & 180.02 & 65.3 \\
6 & 13 & 737.8 & 32.2 & 9 & 174.07 & 71.4 \\
3 & 14 & 262.2 & 67.8 & 3 & 208.72 & 67.5 \\
10 & 15 & 899.9 & 50 & 6 & 204.89 & 63 \\
17 & 16 & 500 & 50 & 6 & 104.29 & 80.9 \\
16 & 17 & 500 & 50 & 6 & 104.28 & 85.9 \\
9 & 18 & 100 & 50 & 6 & 222.48 & 66.7 \\
14 & 19 & 500 & 50 & 11 & 162.92 & 68.8 \\
2 & 20 & 737.8 & 32.2 & 3 & 201.64 & 73.7 \\
& & & & & &
\end{tabular}

THP-CAP-LPs was prepared. The actual values are in close agreement with predicted values regression formula generated for the design in terms of coded factors is

$$
\begin{gathered}
\mathrm{PS}=104.29-5.23 A+6.99 B-5.09 C-1.76 A B-3.85 A C+ \\
4.84 B C+38.68 A^{2}+31.61 B^{2}+23.75 C^{2} \\
\mathrm{EE}=82.67+0.72 A-0.62 B-0.018 C-3.54 A B+0.39 A C+ \\
0.89 B C-6.09 A^{2}-5.29 B^{2}-5.30 C^{2}
\end{gathered}
$$

where, PS and EE are particle size and entrapment efficiency respectively.

\section{Characterization of CAP-LPs and THP-CAP-LPs}

The result obtained by FTIR representing no compatibility issues between the drug and excipients used (Fig. 4a). The developed formulation THP-CAP-LPs was confirmed by using high resolution mass spectrometry (HRMS). The theoretical molecular weight of the formulation was found to be 2316.5 and observed molecular weight of the given formulation was found to be $2316.7[\mathrm{M}+\mathrm{H}]^{+}$(Fig. 3a and b). By dynamic light scattering, it was observed that particle size of THP-CAP-LPs is $148.18 \pm 1.73 \mathrm{~nm}$. When compared to CAP-LPs $(116.20 \pm 1.54$ $\mathrm{nm}$ ), the particle size of THP-CAP-LPs was high which may be due to the surface modification of peptide. Zeta potential of CAP-LPs and THP-CAP-LPs was $-7.8 \mathrm{mV}$ and $3.7 \mathrm{mV}$ respectively (Fig. $4 \mathrm{~b}$ and c). The zeta potential shift may be due to the Michael group ( $\alpha-\beta$ unsaturated group produced when treating with acryloyl chloride) of cholesterol conjugation with THP by a covalent bond formation. This suggests the proper conjugation of the amine group of THP. SEM and TEM studies reported that THP-CAP-LPs was mono-dispersed and spherical shaped (Fig. $4 \mathrm{~d}$ and e). The entrapment efficiency of THP-CAP-LPs and CAP-LPs was found to be $69.76 \pm 0.11 \%$ and $79.23 \pm 0.55 \%$ respectively. The drug loading of THP-CAP-LPs and CAP-LPs was found to be $34.72 \pm 0.11 \%$ and $29.75 \pm 0.44 \%$.

Y1 PARTICLE SIZE
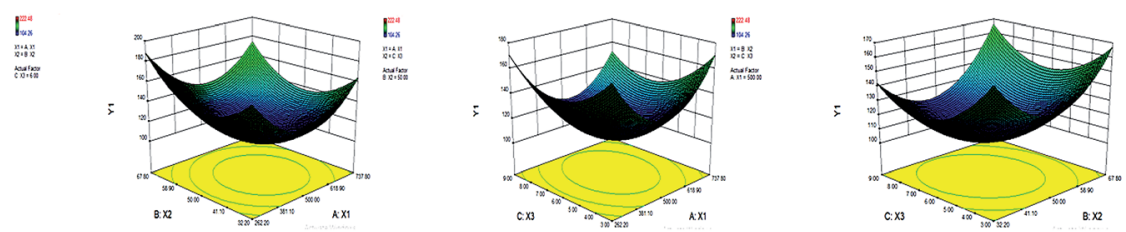

Y2 ENTRAPMENT EFFICIENCY
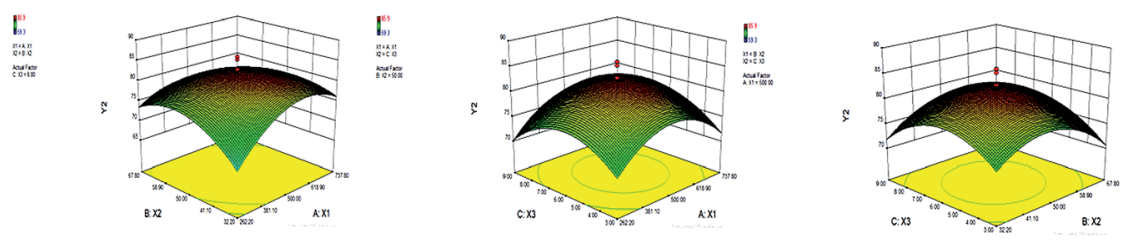

Fig. 1 3D response surface plot screening effect of independent variables (a) cholesterol acrylate concentration (X1) and CAP concentration (X2) (b) cholesterol acrylate concentration (X1) and sonication time (X3) (c) CAP concentration (X2) and sonication time (X3) on particle size (Y1) and entrapment efficiency $(Y 2)$ (note: region in the red represents maxima and region in blue represents minima). 
Table 3 Analysis of particle size (Y1) by using ANOVA

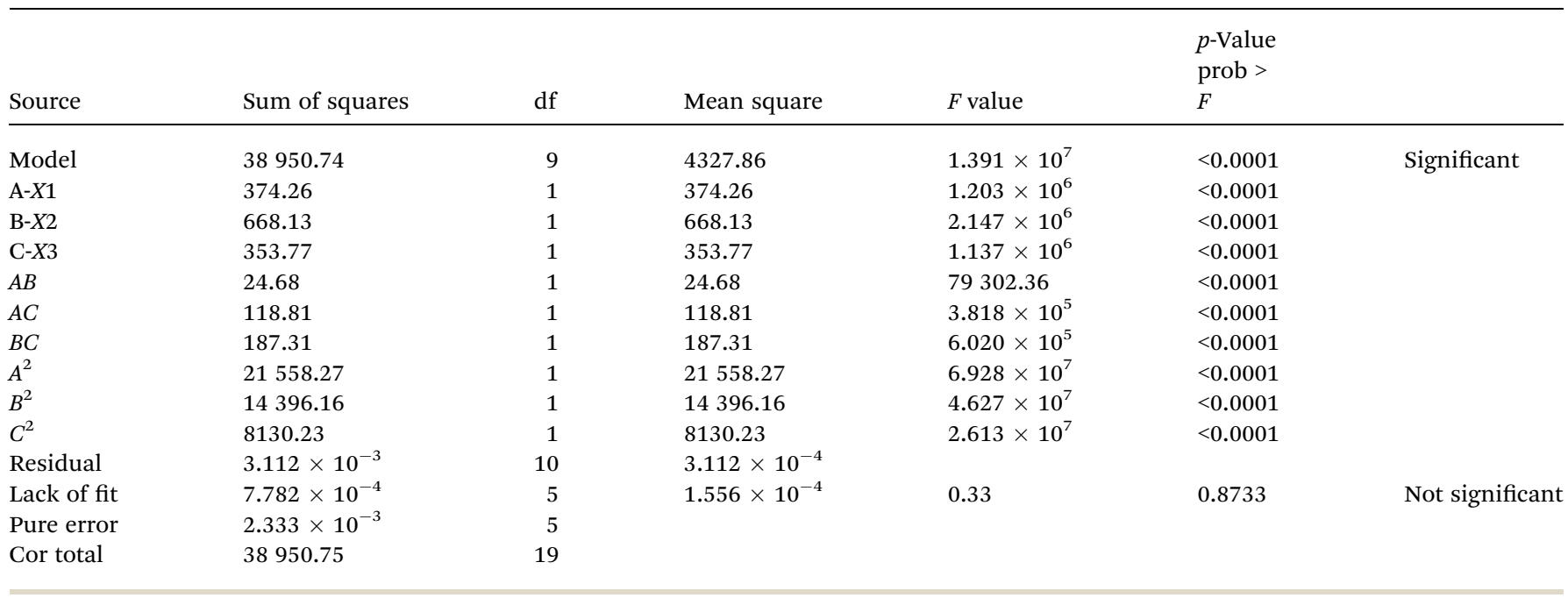

\section{In vitro release of CAP from CAP-LPs and THP-CAP-LPs}

There was no burst release of CAP observed with THP-CAP-LPs and CAP-LPs (Fig. 5). Lipids enhance the rigidity of the encapsulation layers. Surface modification of the lipid can, therefore, alter the flexibility of the lipid layer and delay the rate of drug release. In our study, there is sustained release of drug in THPCAP-LPs when compared to CAP-LPs this may be due to surface modification. Abnormal glycolytic rate, high lactic acid generation and inappropriate drainage by convective transport, $\mathrm{H}^{+}$ ions stack up can be seen in the tumor tissue. So, there will be acidic $\mathrm{pH}$ at tumor site. ${ }^{21}$ To mimic the $\mathrm{pH}$ variation between cellular exterior ( $\mathrm{pH}$ 7.4) and intracellular lysosome ( $\mathrm{pH} 4.5)$, drug release was carried out at different $\mathrm{pH}$ environment. CAP is a weak acid and alkaline in nature is due to the pair of sulfide groups and it shows higher solubility at lower $\mathrm{pH}$. Thereby, the encapsulated CAP in the liposomes has tendency to enter into the release medium of lower $\mathrm{pH}$.

\section{Cellular uptake and cytotoxic activity of CAP, CAP-LPs and THP-CAP-LPs}

Cellular uptake of the THP-CAP-LPs and CAP-LPs was evaluated in MDA-MB453 cells. For cellular uptake imaging studies, the CAP was replaced with Rhodamine 6G in CAP-LPs and THPCAP-LPs to form Rh-LPs and THP-Rh-LPs (Fig. 6). The in vitro cytotoxic activity of CAP, THP-CAP-LPs and CAP-LPs were evaluated by the SRB assay in MDA-MB453 cell line, the cell viability is shown in (Fig. 6). Blank LPs didn't exhibit significant cytotoxicity in MDA-MB 453 cells which confirm the safety of the LPs as carriers. MDA-MB453 cells treated with CAP-LPs and THPCAP-LPs exhibited cytotoxicity at various concentrations $(1.562,3.125,6.25,12.5,25,50,100 \mu \mathrm{M})$. It is evident that CAP, CAP-LPs and THP-CAP-LPs exhibited dose-dependent cytotoxic action. CAP naive drug exhibited lower cytotoxic action when compared to CAP-LPs and THP-CAP-LPs which may be due to the efflux of the diffused drug in the cytoplasm by $\mathrm{P}$ -

Table 4 Analysis of entrapment efficiency (Y2) by using ANOVA

\begin{tabular}{|c|c|c|c|c|c|c|}
\hline Source & Sum of squares & df & Mean square & $F$ value & $\begin{array}{l}p \text {-Value } \\
\text { prob }> \\
F\end{array}$ & \\
\hline Model & 1241.57 & 9 & 137.95 & 17.64 & $<0.0001$ & Significant \\
\hline $\mathrm{A}-X 1$ & 7.14 & 1 & 7.14 & 0.91 & 0.3618 & \\
\hline $\mathrm{B}-X 2$ & 5.17 & 1 & 5.17 & 0.66 & 0.4353 & \\
\hline $\mathrm{C}-X 3$ & $4.480 \times 10^{-3}$ & 1 & $4.480 \times 10^{-3}$ & $5.727 \times 10^{-4}$ & 0.9814 & \\
\hline$A B$ & 100.11 & 1 & 100.11 & 12.80 & 0.0050 & \\
\hline$A C$ & 1.20 & 1 & 1.20 & 0.15 & 0.7034 & \\
\hline$B C$ & 6.30 & 1 & 6.30 & 0.81 & 0.3905 & \\
\hline$A^{2}$ & 533.69 & 1 & 533.69 & 68.22 & $<0.0001$ & \\
\hline$B^{2}$ & 403.28 & 1 & 403.28 & 51.55 & $<0.0001$ & \\
\hline$C^{2}$ & 405.44 & 1 & 405.44 & 51.83 & $<0.0001$ & \\
\hline Residual & 78.23 & 10 & 7.82 & & & \\
\hline Lack of fit & 46.57 & 5 & 9.31 & 1.47 & 0.3412 & Not significant \\
\hline Pure error & 31.66 & 5 & 6.33 & & & \\
\hline Cor total & 1319.80 & 19 & & & & \\
\hline
\end{tabular}




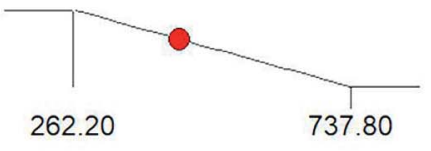

$\mathrm{A}: \mathrm{X} 1=445.44$

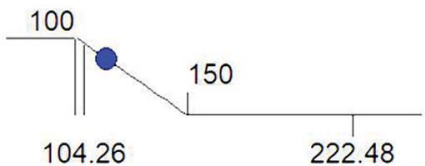

$Y 1=114.036$

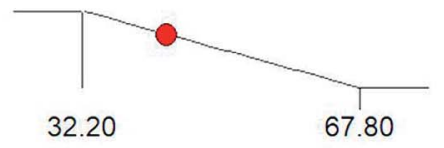

$B: X 2=43.05$

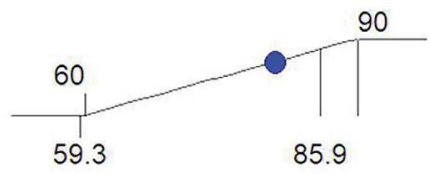

$Y 2=80.8716$

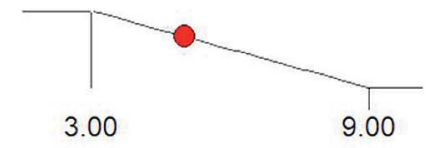

$C: X 3=5.01$

Desirability $=0.677$

Fig. 2 Optimization of the dependent variables particle size (Y1) and entrapment efficiency (Y2) by different concentration of independent variables (X1) cholesterol acrylate (X2) CAP and (X3) sonication time.

glycoprotein (P-gp) pumps. CAP-LPs and THP-CAP-LPs can inhibit P-gp mediated drug efflux (Fig. 7). ${ }^{8-11,22-24}$

\section{THP-CAP-LPs augmented the expression of proapoptotic proteins and inhibited antiapoptotic protein expression}

Apoptosis is generally regulated by the various proapoptotic and antiapoptotic proteins. Generally apoptotic response is regulated by intrinsic apoptotic pathway (mitochondrial mediated) and extrinsic apoptotic pathway. It was reported that CAP induce apoptosis by increasing ROS levels, which is associated with intrinsic apoptotic pathway. We, therefore, observed the expression of various anti-apoptotic and pro-apoptotic proteins of intrinsic apoptotic pathway in MDA-MB453 cells. It was reported that, cancer cells resist apoptosis due to chemotherapeutic agents by upregulation of anti-apoptotic proteins and down regulation of apoptotic proteins. The $\mathrm{Bcl} 2$ family of proteins (Bcl-2, Bcl-xL and $\mathrm{Mcl}-1)$ and survivin are antiapoptotic proteins, which are responsible for chemoresistance. Bax, caspase-3, caspase-9 are some of the examples of pro-apoptotic proteins. $^{25,26}$ THP-CAP-LPs exhibited upregulation of proprotein expressions of caspase-9, and downregulation of antiapoptotic proteins such as $\mathrm{Bcl} 2$ and survivin, when compared with the CAP and CAP-LPs (Fig. 8). ${ }^{27}$

\section{Experimental}

\section{Synthesis of THP-cholesterol conjugate (THP-CL)}

The cholesterol peptide conjugate involves two steps: the solution of acryloyl chloride $(1.3 \mathrm{~mL}, 15.6 \mathrm{mmol})$ in $5 \mathrm{~mL}$ of anhydrous THF was added dropwise to a solution of cholesterol ( $4 \mathrm{~g}$, $10.3 \mathrm{mmol})$ and triethylamine $(4.3 \mathrm{~mL}, 31 \mathrm{mmol})$ in $50 \mathrm{~mL}$ of anhydrous THF at $0{ }^{\circ} \mathrm{C}$ under a positive nitrogen flow. After removing the mixture from ice-bath, allowed to stir for overnight at room temperature. The crude solid was filtered out by using rotary evaporator and redissolved in $50 \mathrm{~mL}$ of DCM and washed with the following solvents like deionized water, hydrochloric acid $(0.5 \mathrm{M})$. The filtrate again extracted by using deionized water and finally neutralized with the sodium bicarbonate $(1 \mathrm{M})$, deionised water and brine solution. The product present DCM was allowed to dry over anhydrous sodium sulphate and pure product was obtained as white solid by using column chromatography (DCM/hexane $1 / 1 \mathrm{v} / \mathrm{v}$ ). The cholesterolacrylate can be conjugated with peptide by using a mixture of DMSO and THF in the presence of a base triethylamine. The above mixture was allowed to stir at $37{ }^{\circ} \mathrm{C}$ for almost 2 days under inert conditions. The pure product was obtained by dialysis using deionized water, followed by freeze-drying.

\section{Preparation of THP-CAP-LPs}

THP-CAP-LPs were prepared by thin film hydration technique by using THP as a lipid in the round bottom flask by using

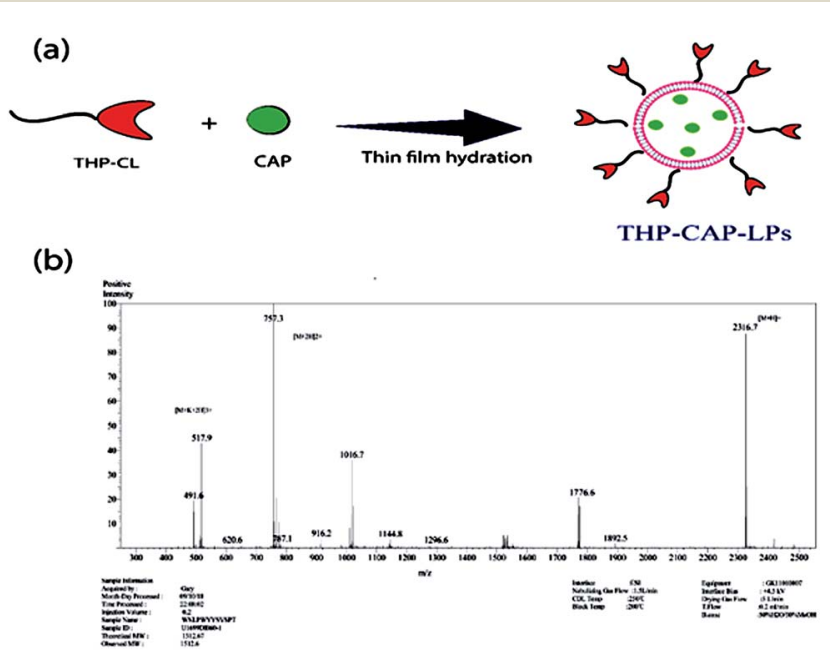

Fig. 3 (a) Digramatic representation of the CAP loaded in the THP-CL to form THP-CAP-LPs by the use of thin film hydration technique. (b) Mass spectrum representing the conformation of the conjugation between the THP and CL. The $[\mathrm{M}+\mathrm{H}]^{+}$peak found at 2316.7 . 
(a)

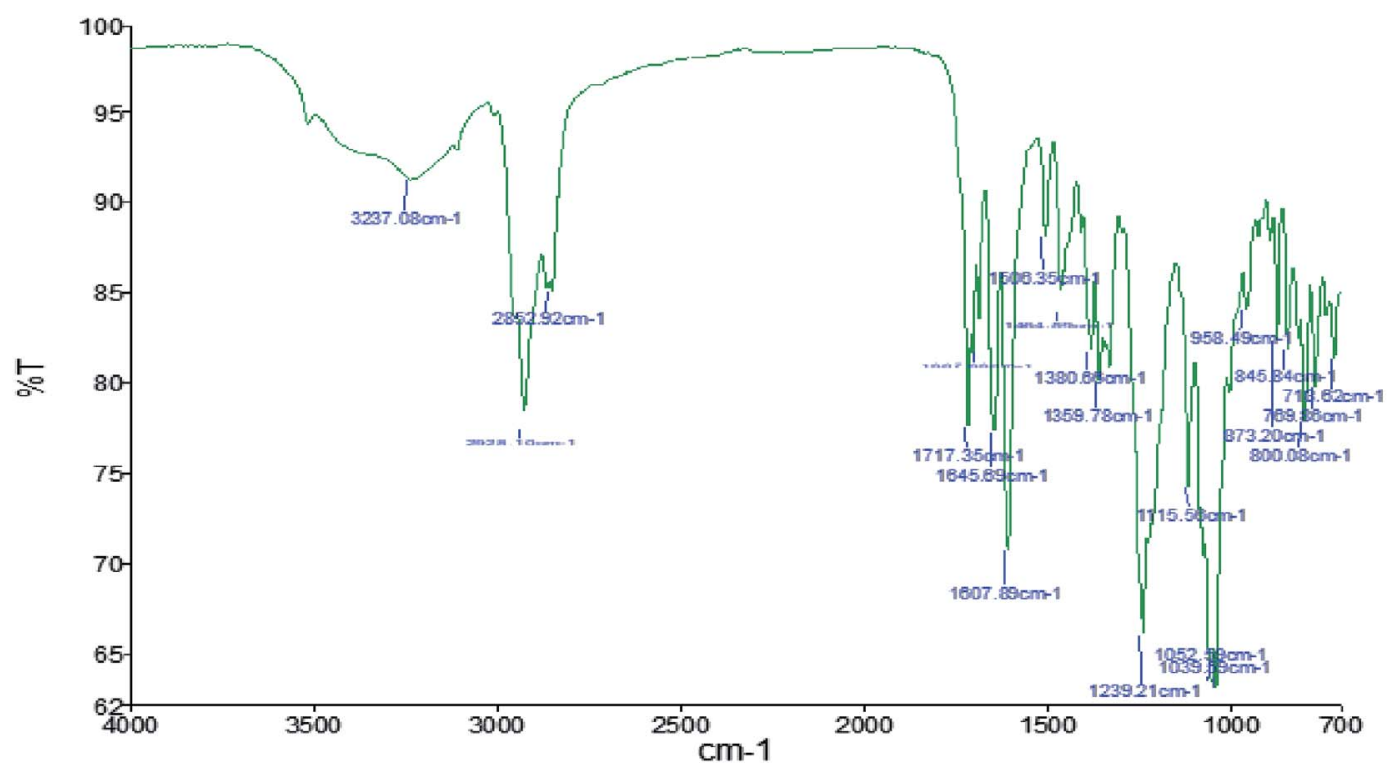

(b)

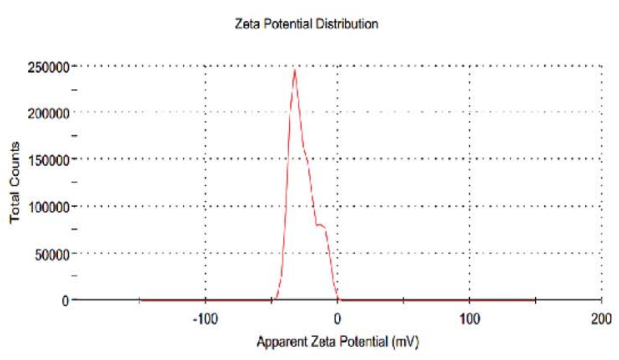

(d)

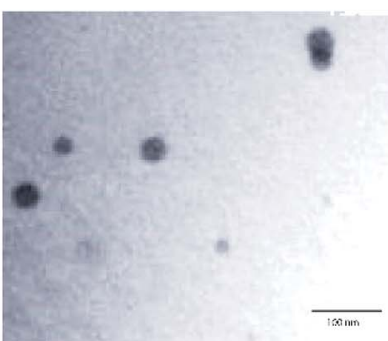

(c)

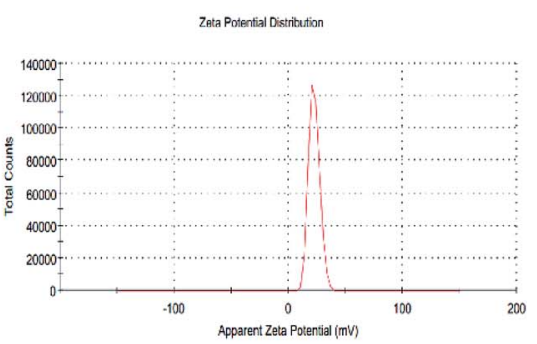

(e)

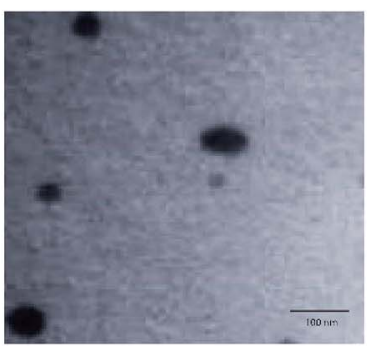

Fig. 4 FT-IR graph (a), zeta potential of CAP-LPs (b) and THP-CAP-LPs (c), TEM images of CAP-LPs (d) and THP-CAP-LPs (e).

organic solvent chloroform and methanol in a ratio of $6: 4$ and kept for evaporation with rotary evaporator by using different parameter such as, vacuum pressure $(160 \mathrm{mmHg}$ to $30 \mathrm{mmHg})$, temperature $\left(35^{\circ} \mathrm{C}\right)$ and rotation speed $(30$ to $40 \mathrm{rpm})$ to get thin film. Afterwards, thin film consist round bottom flask has kept in vacuum oven for overnight at room temperature by using 160 $\mathrm{mmHg}$ vacuum pressure to evaporate remaining chemical residue from the thin film. Then prepared sample further, kept for hydration by using $7.4 \mathrm{pH}$ dihydrogen orthophosphate buffer as hydration medium for 1 to 2 hours at rotary evaporator and further, probe sonication has been done for 3 to 9 minutes to obtain desired particle size. CAP-LPs (non-surface modified LPs) were also prepared by same method except THP was replaced with cholesterol acrylate.

\section{Application of central composite design (CCD) for optimization of THP-CAP-LPs}

For the present study, a 17-run, 3-factor, 3-level CCD was employed to optimize the THP-CAP-LPs using Design-Expert software (Version 8.0.4. Stat-Ease, Inc., 2 Minneapolis, MN). This is a cubic design, 


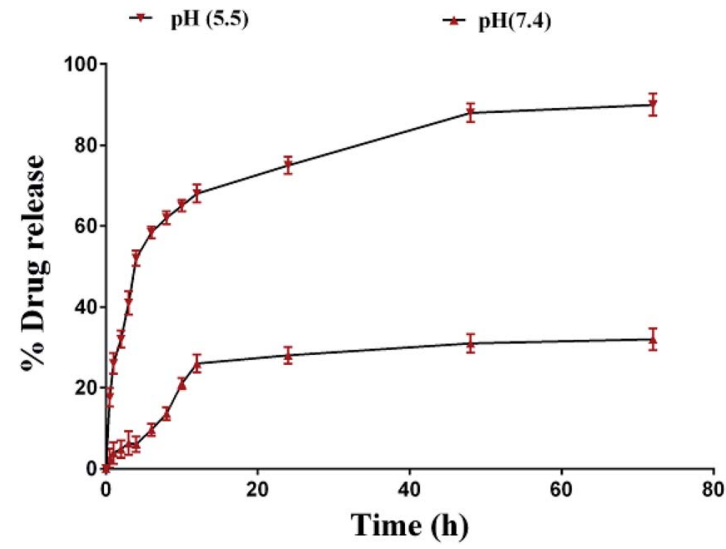

Fig. 5 Drug release profile of THP-CAP-LPs at different time points.

characterized by center point replicates and a set of points at the mid-point of each edge of the multidimensional cube that circumscribe the region of interest. Region of interest aids to investigate the main effects, interaction effects, and quadratic effects of the formulation ingredients and to optimize the formulation. Design matrix with 20 experimental runs was constructed. The amount of THP-CL (X1), CAP (X2), and sonication time $(X 3)$ as the independent variables which were represented by $-1,0$, and +1 , analogous to the low, middle, and high values, respectively as described in Table 1. The model was selected from a quadratic mathematical equation based on significance $(p$ value $<0.05)$ from the model and non-significant lack of fit.

\section{Determination of particle size, zeta potential, and morphology}

The particle size and zeta potential of THP-CAP-LPs and CAPLPs were measured by dynamic light scattering (DLS) Nanotrac Wave ${ }^{\mathrm{TM}}$ (Microtrac, San Diego, CA, USA) before measurement the samples were suspended in deionized water. All measurements were carried out at $250{ }^{\circ} \mathrm{C}$ and performed in triplicate. The morphology of THP-CAP-LPs was analysed using scanning electron microscopy (SEM) and transmission electron microscope (TEM). ${ }^{13,14}$

\section{Determination of drug entrapment efficiency and drug loading}

The amount of CAP in THP-CAP-LPs and CAP-LPs was determined using HPLC. About $10 \mathrm{mg}$ prepared LPs in freeze dried form was dissolved in $10 \mathrm{~mL}$ of $1: 1$ mixture of methanol and sodium dodecyl sulphate, that facilitate the disruption of coat to release the encapsulated drug. Then the mixture was placed in a super filter tube, and then centrifuged by a Sigma-3k30 Centrifuges (Sigma-Aldrich, Seelze, Germany) with $14000 \mathrm{rpm}$ for $10 \mathrm{~min}$ at the temperature of $320^{\circ} \mathrm{C}$. The ultrafiltrate was extracted by methanol, and filtered with a $0.45 \mathrm{~mm}$ filter. The drug concentration in ultrafiltrate was determined as the content of the free drug. ${ }^{15}$ The drug content in the supernatant after centrifugation was measured by HPLC method using an mobile phase delivery pump (LC-20 AD; Shimadzu, Japan) of flow rate $1.2 \mathrm{~mL} \mathrm{~min}^{-1}$, a photodiode array detector (SPDM20A; Shimadzu, Japan) set at $242 \mathrm{~nm}$, a $20 \mu \mathrm{L}$ loop (Rheodyne) and Hypersil C18 $(250 \mathrm{~mm} \times 4.6$ $\mathrm{mm}$ ) was used. The mobile phase consisted of acetonitrile and potassium hydrogen orthophosphate buffer $(\mathrm{pH} 4)$ $(60: 40, \mathrm{v} / \mathrm{v})$. The encapsulation efficiency and loading capacity were calculated by the following equations. The following formulae were used to determine DL (\%) and EE (\%).

DL $(\%)=($ Total amount of drug - Amount of drug in aqueous phase $) /($ Amount of lipid $) \times 100$

EE $(\%)=($ Total amount of drug - Amount of drug in aqueous phase $) /($ Total amount of drug $) \times 100$

\section{Analysis of in vitro drug release}

The in vitro release behaviour of THP-CAP-LPs and CAP-LPs was analysed by performing dialysis bag diffusion method. $1.0 \mathrm{mg}$ of THP-CAP-LPs and CAP-LPs were dispersed in $10 \mathrm{~mL}$ of phosphate buffer saline (PBS) and then transferred into a preswelled dialysis bag with Molecular Weight Cut Off (MWCO) 8-12 kDa (Dialysis membrane-150, HiMedia, Mumbai, India). The bag was individually suspended in $100 \mathrm{~mL}$ of phosphate buffer saline (PBS) pH 4.5 and PBS pH 7.4, at $37^{\circ} \mathrm{C}$ in water bath at $100 \mathrm{rpm}$. Sink condition was maintained by periodically removing $2 \mathrm{~mL}$ sample and replacing equal volume of fresh buffer medium and monitored throughout the release period. The amount of CAP released was determined by an HPLC.
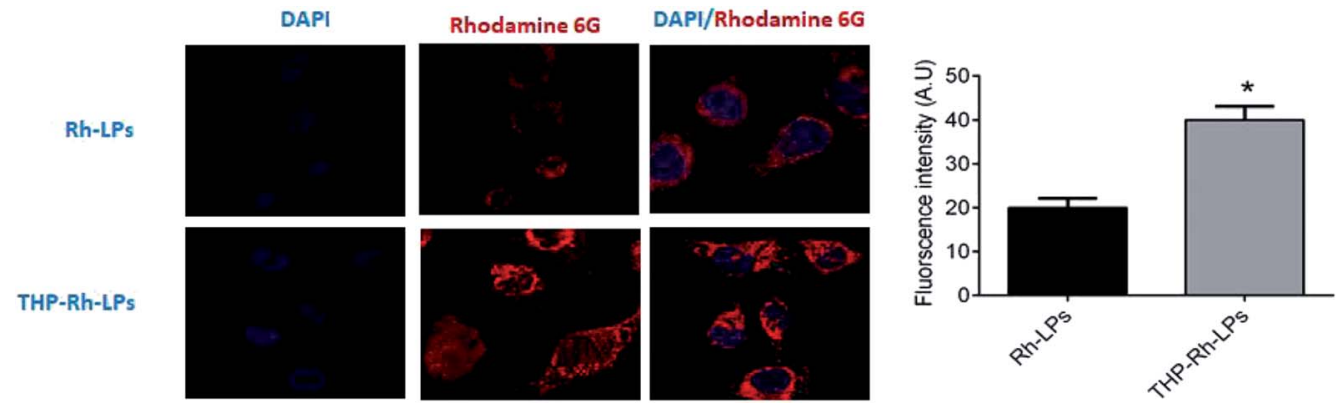

Fig. 6 The cellular uptake of CAP-LPs and THP-CAP-LPs in MDA-MB453 cells. 


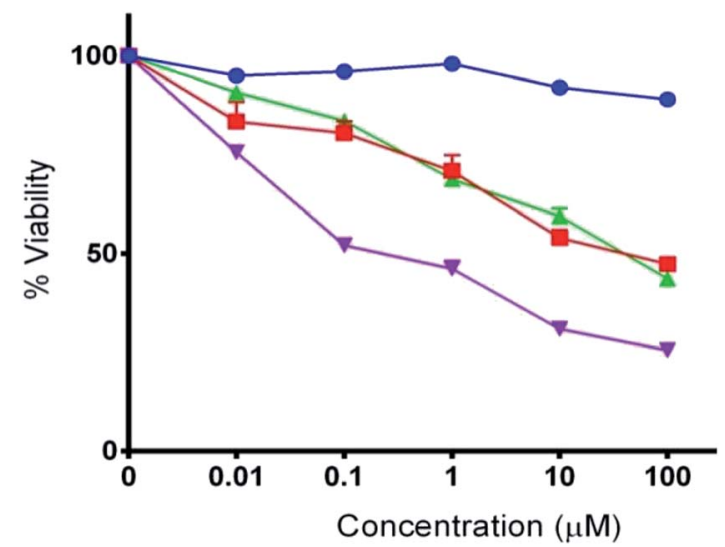

Fig. 7 The in vitro cytotoxic activity of CAP, CAP-LPs and THP-CAPLPS.

\section{Cells and culture conditions}

MDA-MB453 cells were procured from National Center for Cell Science (NCCS), Pune and cultured at humidified atmosphere of $95 \%$ air and $5 \% \mathrm{CO}_{2}$ at $37{ }^{\circ} \mathrm{C}$.

\section{Cellular uptake study}

MDA-MB 453 cells were incubated at $37{ }^{\circ} \mathrm{C}$ with THP-Rh-LPs/RhLPs (equivalent to $1 \mu \mathrm{g} \mathrm{mL} \mathrm{m}^{-1} \mathrm{Rh}$ ) in complete media for $24 \mathrm{~h}$ then the cells were subjected to cytospinning and were fixed with $4 \%$ paraformaldehyde and mounted with DAPI. Cells were then visualized under confocal laser scanning microscopy (Carl Zeiss LSM710, Germany) to determine the cellular uptake. ${ }^{\mathbf{1 6 - 1 8}}$

\section{In vitro cytotoxicity studies}

The cytotoxicity of CAP, THP-CAP-LPs and CAP-LPs against MDA-MB 453 was measured by SRB assay. ${ }^{19}$ Briefly, MDA-MB 453 cells with a density of $\left(1 \times 10^{4}\right.$ per well $)$ were seeded in a 96-well plate and left for incubation for $24 \mathrm{~h}$ under a humidified atmosphere of $95 \%$ air and $5 \% \mathrm{CO}_{2}$ at $370{ }^{\circ} \mathrm{C}$. Then, the cells were treated with serial concentrations $(1.562,3.125,6.25$, 12.5, 25, 50, $100 \mu \mathrm{M}$ ) of CAP, THP-CAP-LPs and CAP-LPs at

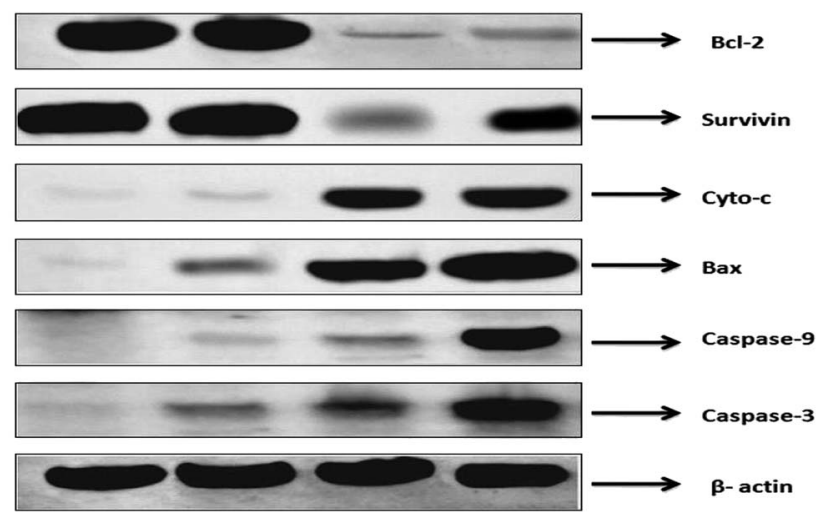

Fig. 8 Western blot analysis of various antiapoptotic and proapoptotic proteins.
$370{ }^{\circ} \mathrm{C}$. The cells were rinsed with cell medium three times and further incubated at $370{ }^{\circ} \mathrm{C}$ for $72 \mathrm{~h}$, followed by fixing of cells with cold trichloroacetic acid, washing and drying in the air. Subsequently, the fixed cells were then stained with $0.4 \%$ SRB dye for $30 \mathrm{~min}$ and the excess dye was washed by $1 \%$ acetic acid. After bound dye dissolved in $10 \mathrm{mM}$ Tris base solution, the absorbance was determined using a Thermo scientific multiscan FC microplate photometer at the wavelength of $540 \mathrm{~nm}$. The data were expressed as the percentages of viable cells compared to the survival of control group (cells treated with medium) and presented as mean \pm SD.

\section{Western blotting}

RIPA buffer (comprising $20 \mathrm{mM} \mathrm{NaH} \mathrm{PO}_{4}, 50 \mathrm{mM} \mathrm{NaF}, 2 \mathrm{mM}$ EDTA, $150 \mathrm{mM} \mathrm{NaCl}, 1 \%$ deoxycholic acid, 0.1\% SDS, pH 7.2) was used to prepare whole cell lysates. Protease inhibitor cocktail (Sigma Aldrich, Mumbai) was used for Western blot analysis. The protein (40 mg) was taken and resolved using 10\% sodium dodecyl sulfate-polyacrylamide gel electrophoresis (SDS-PAGE). Antibodies (anti- $\beta$-actin, anti-Bcl-2, anti-survivin, anti-caspase-9) were selected and obtained from Santacruz biotech and horseradish peroxidase-conjugated goat anti-rabbit IgG was used as secondary antibody. This was later detected using chemiluminescence reagents. Hitachi Gene tools CCDBIO software with Hitachi scanning densitometry was used for determination of relative protein expression. ${ }^{12}$

\section{Statistical analysis}

Statistical analysis was done by performing Student $t$-test. The differences were considered significant for $p$ values of $<0.05$. Data were subjected to statistical analysis using Graphpad prism software (version 6).

\section{Conclusion}

THP-CAP-LPs formulation which was optimized using BoxBehnken experimental design were prepared to overcome the bioavailability problems of CAP and then we have surface modified CAP-LPs with THP to form THP-CAP-LPs. Surface modification of CAP-LPS with THP significantly increased the uptake of CAP in MDA-MB 453 cells. CAP-LPs surface modified with THP promoted targeted therapy, therefore, overcome the bioavailability issues of CAP. THP-CAP-LPs exhibited greater cytotoxic effect when compared to CAP and CAP-LPs, which might be attributed to the ability of surface modification with THP to interfere with intrinsic apoptotic signalling pathways. It was observed that THP-CAP-LPs significantly downregulated anti-apoptotic proteins ( $\mathrm{Bcl} 2$ and survivin), and upregulated pro-apoptotic caspase-9. Thereby we have assumed that the increased apoptotic ability of THP-CAP-LPs may be by interfering intrinsic apoptotic pathway. In conclusion, conjugation of THP with CAP-LPs is a promising targeted delivery of cytotoxic drugs reduces the off-target effects to healthy cells. In addition, THP-CAP-LPs delivery of cytotoxic agents will have improved antitumour activity when compared to either CAP-LPs or CAP alone. However, this study is a preliminary report of in 
vitro results, further targeted delivery to improve antitumour activity need to confirm in vivo in animal models.

\section{Conflicts of interest}

There are no conflicts to declare.

\section{References}

1 C. M. Perou, T. Sørlie, M. B. Eisen, M. van de Rijn, S. S. Jeffrey, C. A. Rees, et al. Molecular portraits of human breast tumours, Nature, 2000, 406(6797), 747-752.

2 J. D. Brenton, L. A. Carey, A. A. Ahmed and C. Caldas, Molecular classification and molecular forecasting of breast cancer: ready for clinical application?, J. Clin. Oncol., 2005, 23(29), 7350-7360.

3 A. K. Smolarek and N. Suh, Chemopreventive activity of vitamin $\mathrm{E}$ in breast cancer: a focus on $\gamma$-and $\delta$-tocopherol, Nutrients, 2011, 3(11), 962-986.

4 D. Peer, J. M. Karp, S. Hong, O. C. Farokhzad, R. Margalit and R. Langer, Nanocarriers as an emerging platform for cancer therapy, Nat. Nanotechnol., 2007, 2(12), 751-760.

$5 \mathrm{~V}$. Torchilin, Tumor delivery of macromolecular drugs based on the EPR effect, Adv. Drug Delivery Rev., 2011, 63(3), 131135.

6 R. Bazak, M. Houri, S. El Achy, S. Kamel and T. Refaat, Cancer active targeting by nanoparticles: a comprehensive review of literature, J. Cancer Res. Clin. Oncol., 2015, 141(5), 769-784.

7 J. D. Byrne, T. Betancourt and L. Brannon-Peppas, Active targeting schemes for nanoparticle systems in cancer therapeutics, Adv. Drug Delivery Rev., 2008, 60(15), 16151626.

8 S. V. Talluri, G. Kuppusamy, V. V. S. R. Karri, K. Yamjala, A. Wadhwani, S. V. Madhunapantula, et al. Application of quality-by-design approach to optimize diallyl disulfideloaded solid lipid nanoparticles, Artif. Cells, Nanomed., Biotechnol., 2016, 1-15.

9 S. Tummala, M. S. Kumar and S. K. Pindiprolu, Improved anti-tumor activity of oxaliplatin by encapsulating in antiDR5 targeted gold nanoparticles, Drug Delivery, 2016, 1-15.

10 S. K. S. Pindiprolu, P. K. Chintamaneni, P. T. Krishnamurthy and K. Ratna Sree Ganapathineedi, Formulationoptimization of solid lipid nanocarrier system of STAT3 inhibitor to improve its activity in triple negative breast cancer cells, Drug Dev. Ind. Pharm., 2019, 45(2), 304-313.

11 X.-G. Zhang, J. Miao, Y.-Q. Dai, Y.-Z. Du, H. Yuan and F.-Q. Hu, Reversal activity of nanostructured lipid carriers loading cytotoxic drug in multi-drug resistant cancer cells, Int. J. Pharm., 2008, 361(1), 239-244.

12 S. Tummala, K. Gowthamarajan and M. Satish Kumar, Oxaliplatin immunohybrid nanoparticles in vitro synergistic suppression evaluation in treatment of colorectal cancer, Artif. Cells, Nanomed., Biotechnol., 2016, 1-9.

13 T. Venkata Siddhartha, V. Senthil, I. Sai Kishan, R. Basha Khatwal and S. V. Madhunapantula, Design and development of oral nanoparticulated insulin in multiple emulsion, Curr. Drug Delivery, 2014, 11(4), 472-485.

14 S. K. S. Pindiprolu, P. T. Krishnamurthy, P. K. Chintamaneni and V. V. S. R. Karri, Nanocarrier based approaches for targeting breast cancer stem cells, Artif. Cells, Nanomed., Biotechnol., 2018, 46(5), 885-898.

15 F.-Q. Hu, S.-P. Jiang, Y.-Z. Du, H. Yuan, Y.-Q. Ye and S. Zeng, Preparation and characterization of stearic acid nanostructured lipid carriers by solvent diffusion method in an aqueous system, Colloids Surf., B, 2005, 45(3), 167-173.

16 L. Zhang, D. Zhu, X. Dong, H. Sun, C. Song, C. Wang, et al. Folate-modified lipid-polymer hybrid nanoparticles for targeted paclitaxel delivery, Int. J. Nanomed., 2015, 10, 2101.

17 G. Sharma, J. Park, A. R. Sharma, J.-S. Jung, H. Kim, C. Chakraborty, et al. Methoxy Poly (ethylene glycol)-Poly (lactide) Nanoparticles Encapsulating Quercetin Act as an Effective Anticancer Agent by Inducing Apoptosis in Breast Cancer, Pharm. Res., 2015, 32(2), 723-735.

18 T. H. Tran, A. N. Nguyen, J. O. Kim, C. S. Yong and C. N. Nguyen, Enhancing activity of artesunate against breast cancer cells via induced-apoptosis pathway by loading into lipid carriers, Artif. Cells, Nanomed., Biotechnol., 2016, 44(8), 1979-1987.

19 V. Vichai and K. Kirtikara, Sulforhodamine B colorimetric assay for cytotoxicity screening, Nat. Protoc., 2006, 1(3), 1112-1116.

20 R. Chen, S. Wang, J. Zhang, M. Chen and Y. Wang, Aloeemodin loaded solid lipid nanoparticles: formulation design and in vitro anti-cancer study, Drug Delivery, 2014, 1-9.

21 P. Vaupel, F. Kallinowski and P. Okunieff, Blood flow, oxygen and nutrient supply, and metabolic microenvironment of human tumors: a review, Cancer Res., 1989, 49(23), 64496465.

22 S. Majumdar and A. K. Mitra, Chemical modification and formulation approaches to elevated drug transport across cell membranes, Expert Opin. Drug Delivery, 2006, 3(4), 511-527.

23 M. W. Nasser, N. A. Wani, D. K. Ahirwar, C. A. Powell, J. Ravi, M. Elbaz, et al. RAGE mediates S100A7-induced breast cancer growth and metastasis by modulating the tumor microenvironment, Cancer Res., 2015, 75(6), 974-985.

24 A. Riehl, J. Németh, P. Angel and J. Hess, The receptor RAGE: Bridging inflammation and cancer, Cell Commun. Signaling, 2009, 7(1), 12.

25 P. Elumalai, D. Gunadharini, K. Senthilkumar, S. Banudevi, R. Arunkumar, C. Benson, et al. Induction of apoptosis in human breast cancer cells by nimbolide through extrinsic and intrinsic pathway, Toxicol. Lett., 2012, 215(2), 131-142.

26 A. S. Gillings, K. Balmanno, C. M. Wiggins, M. Johnson and S. J. Cook, Apoptosis and autophagy: BIM as a mediator of tumour cell death in response to oncogene-targeted therapeutics, FEBS J., 2009, 276(21), 6050-6062.

27 J.-E. Lee, R.-A. Lee, K.-H. Kim and J.-H. Lee, Induction of apoptosis with diallyl disulfide in AGS gastric cancer cell line, J. Korean Surg. Soc., 2011, 81(2), 85-95. 